\title{
THERMAL ASYMMETRY ANALYSIS OF MOTORIZED SPINDLES
}

\author{
L. Koch ${ }^{1 *}$, N. Steinbock ${ }^{1}$, G. Krueger ${ }^{1}$ \\ ${ }^{1}$ University of Applied Sciences Wuerzburg-Schweinfurt, Laboratory of Machine Tools, Schweinfurt, Germany \\ *Corresponding author; e-mail: Lukas.koch@fhws.de
}

\begin{abstract}
This paper presents a method to quantify and reduce thermal asymmetry of motorized spindles. Thermal asymmetry leads to angular and radial deflections at the tool center point. In contrast to simple thermal expansion issues, these effects are harder to compensate. Therefore, the causes of the asymmetries should preferably be evident in the construction phase. This paper introduces a newly developed mathematical formulation to quantify thermal asymmetry. Thermal asymmetry is observed along the longitudinal axis of a motorized spindle. The formulation quantifies thermal asymmetries as a difference of a geometrical centroid and a newly introduced thermal centroid. For this analysis, several motor spindles with different fluid cooling circulation systems were observed. In order to show the legitimacy of the formulation, the spindle's calculated thermal asymmetries are compared with their respective radial tool center point displacements. The results show that the asymmetries correlate with the displacements. Furthermore, the quantification of the thermal asymmetry actually allows to locate its causes. In motor spindles the asymmetry is mostly caused by the complex fluid circulation system. The spindle with the worst cooling circulation showed a radial displacement of $26,32 \mu \mathrm{m}$. Through thermal asymmetry optimization of the circulation's heat transfer, the displacement could be reduced to $0,66 \mu \mathrm{m}$. The developed method is not limited to motorized spindles. It will be investigated further to develop a generally valid formulation.
\end{abstract}

Keywords:

Motor spindle; thermal asymmetry; electric motor; machine tools

\section{INTRODUCTION}

Manufacturing precision is still one of the most important requirement for machine tools. However, precision is reduced by the displacement of the tool center point (TCP). TCP displacement has geometrical, static, dynamic and thermal causes [Putz 2019], with thermal errors being the dominant influence, accounting for about $50 \%$ [Weck 1995], $57 \%$ [Putz 2018] or even up to $75 \%$ [Mayr 2012] of the total displacement.

Many recent studies have focused on compensating thermal displacement through different control-sided approaches. The compensation strategies are often based on machine parameters [Putz 2016, Brecher 2015, Naumann 2016], deformation sensors [Baum 2018, Brecher 2017] or temperature sensors [Brecher 2009, Brecher 2014]. These approaches try to determine a correlation between the respective sensor values / machine parameters and the TCP displacement. Obviously, these methods require timeconsuming calibration measures with expensive TCP displacement measuring devices and therefore see little use in practice. In contrast to these approaches without cause-effect knowledge, structure model based approaches [Beitelschmidt 2015, Thiem 2016, Thiem 2017] use actual physical/ structural models to simulate the deformation during machining. The compensation of TCP displacement is accomplished by calculating the thermomechanical cause-effect relation. However, these approaches also remain problematic, as the boundary conditions of the simulation models often lack the required accuracy [Grossmann 2015]. While these strategies seem to be an effective way to compensate thermal expansion, they usually perform worse when it comes to thermally induced deflection [Brecher 2009, Zhang 2018, Brecher 2017]. Thermal deflection leads to angular errors at the TCP and conventional machining centers without rotatory axis cannot compensate these errors at all [Grossmann 2006].

The objective of this paper is to analyze the causes of these thermally induced deflections and angular errors with a newly developed quantification approach. Once the causes of thermal deflection are identified, the problem of thermal deflection can be solved without a complex control-sided compensation. Fig. 1 visualizes the effect chain of thermally induced TCP displacement in machine tools. In contrast to an earlier concept by Brecher [Brecher 2014], the effect chain of thermal displacement is examined dividedly in this paper. TCP displacement is caused by a superposition of both thermal expansion and thermal deflection problems. Thermal expansion results from rising temperatures. As 


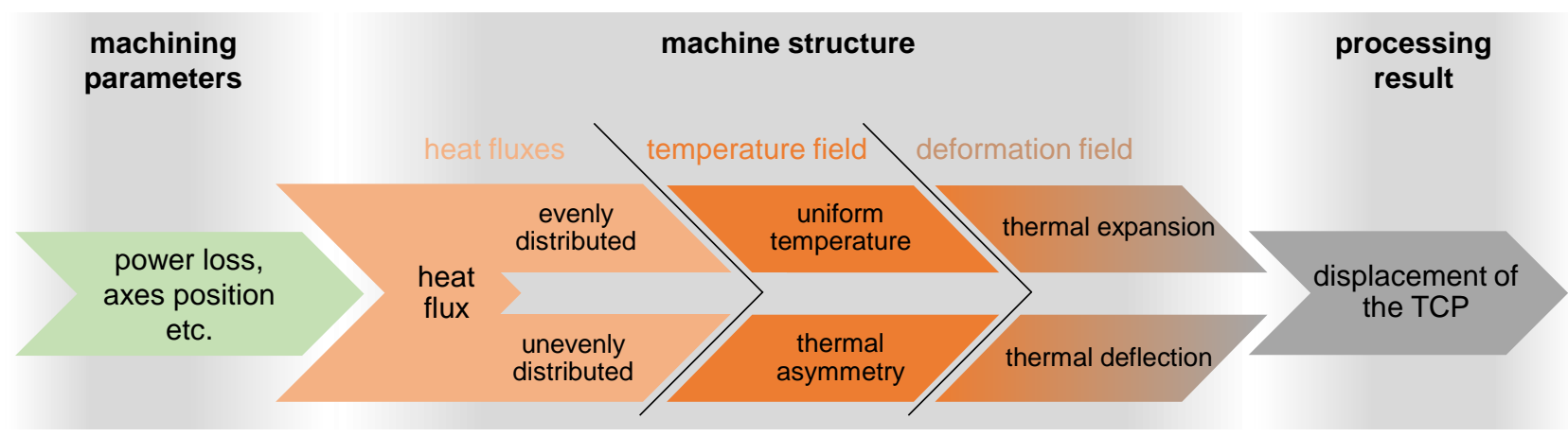

Fig. 1: Divided effect chain of thermally induced deflections in machine tools. The TCP displacement is a superposition of thermal expansion and deflection problems.

long as the temperature field is uniform, no deflection occurs. However, as soon as the heat fluxes are distributed unevenly, the temperature field becomes asymmetrical. This thermal asymmetry is the cause of thermal deflection.

Thermal asymmetry is commonly regarded as an essential machine design criterion [Brecher 2018, Horejs 2012, Liu 2016, Koch 2017, Abele 2010], although no clear definition of the term exists. This is problematic, especially for manufacturers of motorized spindles with highest precision requirements. For this reason, a method to compute thermal asymmetry is presented in this paper. This computation allows to locate the causes of thermal asymmetry, thereby tackling the issue within the construction phase. Solved thermal asymmetry issues reduce TCP displacement problems to thermal expansion issues. Therefore, this new strategy complements any control-sided TCP displacement compensation approach.

\section{QUANTIFICATION OF THERMAL ASYMMETRY}

The following formulation was originally developed to describe the thermal asymmetry of motorized spindles. Therefore, the principle to quantify it will be derived based on a cylindrical component similar to motorized spindles. For a thermal asymmetry analysis, a suitable data basis is necessary. For this reason, using finite element models is recommended. Thermal asymmetry requires a separate analysis of:

- The component's geometry (chapter 2.2) and

- $\quad$ its temperature field (chapter 2.3).

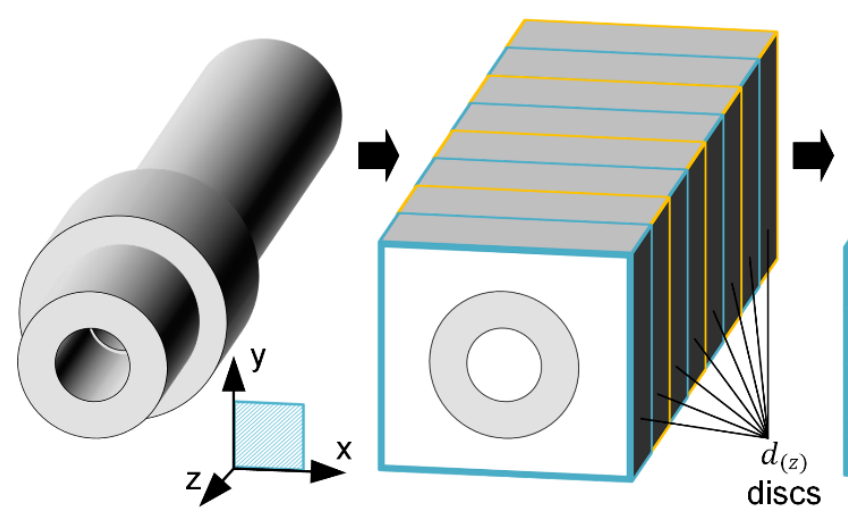

(a) observed component (b) slicing the component in $d_{(z)}$ discs

\subsection{Spatial discretization}

The individual node distribution of the underlying finite element mesh is unknown. Thus, the quantification of the geometrical and thermal constitution requires a separate discretization of the component. This separate discretization establishes a less mesh dependent result. Areas with heterogeneous node distribution are treated equivalently. This is achieved by dividing the component into equivalently sized volume elements.

First of all, the analysis requires a suitable coordinate system (see Fig. 2 a)). Using a Cartesian coordinate system is recommended. Thermal asymmetry observations based on Cartesian coordinate systems are better suited for graphical result processing. The graphical processing allows the location of the asymmetry's cause. Due to space problems, the graphical processing is published in a subsequent paper.

The position of the coordinate system is irrelevant for this method. Thermal asymmetry is calculated for planes along an axis. In the example, thermal asymmetry is observed along the z-axis. Therefore, the component needs to be divided into a finite number of discs $d_{(z)}$ along the z-axis, as displayed in Fig. 2 b). Each of these discs is then further subdivided into a finite number $i$ of volumes $V_{i}$ (Fig. $2 \mathrm{c}$ )). Both the number of discs and the number of volumes should be varied based on the geometry and temperature field complexity of the observed component.

\subsection{Geometrical description}

In order to describe the component geometrically, the centroid of each disc is calculated. The geometrical centroid of any system of composed volumes $V_{i}$ can be calculated based on equation (1) [Fetzer 1999]. $\boldsymbol{r}^{[g]}$ is a column vector

Fig. 2: Discretization method and exemplary observation of six volumes. 
with the three spatial coordinates $\left(x^{[g]}, y^{[g]}\right.$ and $\left.z^{[g]}\right) . \boldsymbol{r}_{i}$ is the vector which represents the spatial position of each individual volume $V_{i}$.

$\boldsymbol{r}^{[g]}=\frac{\sum_{i}\left(\boldsymbol{r}_{i} \cdot V_{i}\right)}{\sum_{i} V_{i}}$

The advantage of this approach is the possibility to represent holes in the geometry with empty volumes $V_{i}$. Therefore, the observation of complex geometries is possible with accordingly sized volume elements.

Equation (1) is then applied to the quantification example in Fig. 2 along the z-axis with discs $d_{(z)}$. The problem becomes two dimensional. Each discs' $d_{(z)}$ geometrical centroid location is calculated with equation (2) and (3). Equation (2) represents the calculation of the centroid's $x$ coordinate and equation (3) the quantification of its $y$ coordinate. $x_{i}$ and $y_{i}$ are the coordinates of each volumes $V_{i}$ centroid.

$x_{d(z)}^{[g]}=\frac{\sum_{i}\left(x_{i} \cdot V_{i}\right)}{\sum_{i} V_{i}}$

$y_{d(z)}^{[g]}=\frac{\sum_{i}\left(y_{i} \cdot V_{i}\right)}{\sum_{i} V_{i}}$

In order to facilitate the calculation of complex geometries a simplification is introduced based on Fig. $2 \mathrm{~d}$ ), which represents six of the volumes $V_{i}$ in Fig. $2 \mathrm{c}$ ). The following equation (4) is equation (3) applied to the example in Fig. 2 d). $V_{32}$ is empty and should therefore be treated as blank volume. Instead of observing each volume individually as in equation (4), a row-based calculation is introduced with equation (5). The rows' $\chi$ distance $x_{d(z)}^{(\chi)}$ (Fig. $2 \mathrm{~d})$ ) is identical for two of the volumes $\left(V_{11}\right.$ and $V_{12}$ etc.) and thereby the calculation is simplified. Furthermore, all volume elements $V_{i}$ are identically in size and the equation is reduced accordingly (equation (6)).

$x_{d(z)}^{[g]}=\frac{x_{1} \cdot V_{11}+x_{2} \cdot V_{12}+x_{3} \cdot V_{21}+x_{4} \cdot V_{22}+x_{5} \cdot V_{31}+x_{6} \cdot V_{32}}{V_{11}+V_{12}+V_{21}+V_{22}+V_{31}+V_{32}}$

$=\frac{x_{1} \cdot V_{11}+x_{2} \cdot V_{12}+x_{3} \cdot V_{21}+x_{4} \cdot V_{22}+x_{5} \cdot V_{31}+x_{6} \cdot 0}{V_{11}+V_{12}+V_{21}+V_{22}+V_{31}+0}$

$x_{d(z)}^{[g]}=\frac{x_{d(z)}^{(1)} \cdot\left(V_{11}+V_{12}\right)+x_{d(z)}^{(2)} \cdot\left(V_{21}+V_{22}\right)+x_{d(z)}^{(3)} \cdot V_{31}}{V_{11}+V_{12}+V_{21}+V_{22}+V_{31}}$

$x_{d(z)}^{[g]}=\frac{2 \cdot x_{d(z)}^{(1)}+2 \cdot x_{d(z)}^{(2)}+x_{d(z)}^{(3)}}{5}$

In order to evaluate larger geometries, the formulation is developed further to equation (7), which features an arbitrary number of volumes. Each row $\chi$ is represented by their number of volumes $n_{d(z)}^{(\chi)}$ and the number of empty volumes $n_{d(z)}^{(\chi, 0)}$. The denominator represents the difference between the number of all volumes $N_{d(z)}$ and the number of empty volumes $N_{d(z)}^{(0)}$ of each disc $d_{(z)}$.

$x_{d(z)}^{[g]}=\frac{\left(n_{d(z)}^{(1)}-n_{d(z)}^{(1,0)}\right) \cdot x_{d(z)}^{(1)}+\left(n_{d(z)}^{(2)}-n_{d(z)}^{(2,0)}\right) \cdot x_{d(z)}^{(2)}+\left(n_{d(z)}^{(3)}-n_{d(z)}^{(3,0)}\right) \cdot x_{d(z)}^{(3)}}{N_{d(z)}-N_{d(z)}^{(0)}}(7)$

In the last step, this equation is enhanced to feature an arbitrary number of rows $\chi$ (equation (8)). Unlike the previous equations, this variant is capable of calculating the geometrical centroids $\mathrm{x}$-coordinate of the whole disc in Fig. 2 c). Analogously, the respective y-coordinate can be calculated with equation (9). This formulation is based on rows $\gamma$ in y-direction. Although the observed disc is the same, the set of observed rows $\gamma$ changes just as the number of volumes $n_{d(z)}^{(\gamma)}$ and empty volumes $n_{d(z)}^{(\gamma, 0)}$ per row. The denominator remains identical to the formulation in $\mathrm{x}$ direction. $x_{d(z)}^{[g]}=\frac{\sum_{\chi}\left[x_{d(z)}^{(x)} \cdot\left(n_{d(z)}^{(\chi)}-n_{d(z)}^{(x, 0)}\right)\right]}{N_{d(z)}-N_{d(z)}^{(0)}}$

$y_{d(z)}^{[g]}=\frac{\sum_{\gamma}\left[y_{d(z)}^{(\gamma)} \cdot\left(n_{d(z)}^{(\gamma)}-n_{d(z)}^{(\gamma, 0)}\right)\right]}{N_{d(z)}-N_{d(z)}^{(0)}}$

\subsection{Thermal description}

The quantification of thermal asymmetry requires a second parameter. This parameter has to be capable of determining the thermal condition of the observed component. For this reason, a so-called thermal centroid is introduced. Just as the calculation of the geometrical centroid the thermal centroids calculation is based on the generally valid form to calculate centroids of composed volumes (equation (1)). However, as displayed in equation (10), the volume is replaced with its average temperature $\bar{T}_{i}$ to calculate the position of the thermal centroid $\boldsymbol{r}^{[t]}$. Wherein $\boldsymbol{r}_{i}$ does still represent the spatial position of each volume element $V_{i}$.

$\boldsymbol{r}^{[t]}=\frac{\sum_{i}\left(\boldsymbol{r}_{i} \cdot \bar{T}_{i}\right)}{\sum_{i} \bar{T}_{i}}$

The average volume element temperature $\bar{T}_{i}$ can be calculated after assigning each node temperature to the respective volume element (equation (11)). $\bar{T}_{i}$ is calculated as the arithmetic average of the nodes' $j$ temperatures which were assigned to the respective volume element $V_{i}$. $J$ is the total number of nodes in each volume. Herein the issues of inhomogeneous meshes becomes visible. Areas with higher node densities have a higher influence on the result of equation (11). Therefore, an appropriate sizing of the volume elements is essential, as this strategy deceases the effect of inhomogeneous meshes. Identically sized volume elements furthermore assure that this issue is regarded constantly for the whole component. Alternatively, homogenous meshes have a comparable effect.

$\bar{T}_{i}=\frac{1}{J} \cdot \sum_{j} T_{j}$

Just like in the previous chapter, this derivation is based on Fig. $2 \mathrm{~d}$ ). Therefore, the problem becomes two dimensional and equation (10) can be simplified to the following equation (12) and (13). Equation (12) calculates the $x$ coordinate of the thermal centroid $x_{d(z)}^{[t]}$ and equation (13) the $y$-coordinate $y_{d(z)}^{[t]} . x_{i}$ and $y_{i}$ still represent the volume elements $\mathrm{x}$ - and $\mathrm{y}$-coordinate.

$x_{d(z)}^{[t]}=\frac{\sum_{i}\left(x_{i} \cdot \bar{T}_{i}\right)}{\sum_{i} \bar{T}_{i}}$

$y_{d(z)}^{[t]}=\frac{\sum_{i}\left(y_{i} \cdot \bar{T}_{i}\right)}{\sum_{i} \bar{T}_{i}}$

These equations are simplified just like the equations (2) and 3 for the geometrical description. Based on Fig. 2 d), equation (14) shows the row-based quantification of the thermal centroid. It is derived analogically to equation (6). The only difference are the temperatures of the volume elements rows $\chi\left(\bar{T}_{d(z)}^{(1)}, \bar{T}_{d(z)}^{(2)}, \bar{T}_{d(z)}^{(3)}\right)$ which cannot be reduced any further. They are calculated through averaging the volume element $i$ temperatures $\bar{T}_{i, \chi}$ of each row $\chi$ (see equation (15)).

$x_{d(z)}^{[t]}=\frac{2 \cdot x_{d(z)}^{(1)} \cdot \bar{T}_{d(z)}^{(1)}+2 \cdot x_{d(z)}^{(2)} \cdot \bar{T}_{d(z)}^{(2)}+x_{d(z)}^{(3)} \cdot \bar{T}_{d(z)}^{(3)}}{2 \cdot \bar{T}_{d(z)}^{(1)}+2 \cdot \bar{T}_{d(z)}^{(2)}+\bar{T}_{d(z)}^{(3)}}$

$\bar{T}_{d(z)}^{(\chi)}=\frac{1}{n_{d(z)}^{(\chi)}-n_{d(z)}^{(\chi, 0)}} \cdot \sum_{i, \chi} \bar{T}_{i, \chi}$

Furthermore, equation (14) should feature an arbitrary number of volumes. This is achieved by multiplying with the 
difference of the number of all volumes $n_{d(z)}^{(\chi)}$ and the number of empty volumes $n_{d(z)}^{(\chi, 0)}$ of each row $\chi$ (equation (16)). This form does also require the calculation of an average temperature of the whole disc $\bar{T}_{d(z)}$. It is calculated as the arithmetic average of the volume elements of the disc $d_{(z)}$, where $\bar{T}_{i, d(z)}$ is the average temperature of each volume element $i$ of the respective disc (equation (17)).

$x_{d(z)}^{[t]}=$

$\frac{\left(n_{d(z)}^{(1)}-n_{d(z)}^{(1,0)}\right) \cdot x_{d(z)}^{(1)} \cdot \bar{T}_{d(z)}^{(1)}+\left(n_{d(z)}^{(2)}-n_{d(z)}^{(2,0)}\right) \cdot x_{d(z)}^{(2)} \cdot \bar{T}_{d(z)}^{(2)}+\left(n_{d(z)}^{(3)}-n_{d(z)}^{(3,0)}\right) \cdot x_{d(z)}^{(3)} \cdot \bar{T}_{d(z)}^{(3)}}{\bar{T}_{d(z)} \cdot\left(N_{d(z)}-N_{d(z)}^{(0)}\right)}$

$\bar{T}_{d(z)}=\frac{1}{N_{d(z)}-N_{d(z)}^{(0)}} \cdot \sum_{i, d(z)} \bar{T}_{i, d(z)}$

The generally valid form in equation (18) is derived through an arbitrary number of rows $\chi$. Equation (19) for the therma centroid calculation in y-direction is derived analogously. Just like in equation (9), $\gamma$ represents the rows in $y$ direction. The average row temperature $\bar{T}_{d(z)}^{(\gamma)}$ is calculated analogously to equation (15).

$x_{d(z)}^{[t]}=\frac{\sum_{\chi}\left[x_{d(z)}^{(\chi)} \cdot \bar{T}_{d(z)}^{(\chi)} \cdot\left(n_{d(z)}^{(\chi)}-n_{d(z)}^{(\chi, 0)}\right)\right]}{\bar{T}_{d(z)} \cdot\left(N_{d(z)}-N_{d(z)}^{(0)}\right)}$

$y_{d(z)}^{[t]}=\frac{\sum_{\gamma}\left[x_{d(z)}^{(\gamma)} \cdot \bar{T}_{d(z)}^{(\gamma)} \cdot\left(n_{d(z)}^{(\gamma)}-n_{d(z)}^{(\gamma, 0)}\right)\right]}{\bar{T}_{d(z)} \cdot\left(N_{d(z)}-N_{d(z)}^{(0)}\right)}$

\subsection{Thermal asymmetry calculation}

The quantification of the geometrical and thermal centroid locations makes it possible to calculate the thermal asymmetry. This is achieved by quantifying therma asymmetry for a disk (see Fig. 2 c)). Based on that, a formulation is introduced to calculate the thermal asymmetry of the whole geometry (Fig. 2 b)).

\section{Thermal asymmetry of discs}

Based on the geometrical and the thermal centroid calculations, the thermal asymmetry of each disc $d_{(z)}$ can be calculated as the difference of the thermal centroid (equation (18) and (19)) and the geometrical centroid (equation (8) and (9)). Equation (20) and (21) represent the corresponding formulations for the $x$-direction and $y$ direction. Therefore, thermal asymmetries $\left(x_{d(z)}, y_{d(z)}\right)$ are defined as the displacement of the thermal centroid relatively to the geometrical centroid. Based on that, thermal asymmetry terms $\left(x_{d(z)}, y_{d(z)}\right)$ are a distance or length and their unit is $\mathrm{m}$.

$x_{d(z)}=\frac{\sum_{\chi}\left[x_{d(z)}^{(\chi)} \cdot \bar{T}_{d(z)}^{(\chi)} \cdot\left(n_{d(z)}^{(\chi)}-n_{d(z)}^{(\chi, 0)}\right)\right]}{\bar{T}_{d(z)} \cdot\left(N_{d(z)}-N_{d(z)}^{(0)}\right)}-\frac{\sum_{\chi}\left[x_{d(z)}^{(\chi)} \cdot\left(n_{d(z)}^{(\chi)}-n_{d(z)}^{(\chi, 0)}\right)\right]}{N_{d(z)}-N_{d(z)}^{(0)}}$

$y_{d(z)}=\frac{\sum_{\gamma}\left[x_{d(z)}^{(\gamma)} \cdot \bar{T}_{d(z)}^{(\gamma)} \cdot\left(n_{d(z)}^{(\gamma)}-n_{d(z)}^{(\gamma, 0)}\right)\right]}{\bar{T}_{d(z)} \cdot\left(N_{d(z)}-N_{d(z)}^{(0)}\right)}-\frac{\sum_{\gamma}\left[y_{d(z)}^{(\gamma)} \cdot\left(n_{d(z)}^{(\gamma)}-n_{d(z)}^{(\gamma, 0)}\right)\right]}{N_{d(z)}-N_{d(z)}^{(0)}}$

If the observed disc has a homogenous temperature field both the thermal and geometrical centroids have the same location. Accordingly, the thermal asymmetries $x_{d(z)}, y_{d(z)}$ are zero. In other words, the observed disc is thermosymmetrical. As soon as the thermal and geometrical centroid are not congruent $\left(x_{d(z)} \neq 0\right.$ and/or $\left.y_{d(z)} \neq 0\right)$ anymore, the observed disc is thermo-asymmetrical.

\section{Thermal asymmetry of the whole geometry}

To evaluate the thermal asymmetry of a component as a whole, the results of the individual discs' calculations in equation 20 and 21 need to be cumulated. The direction of the individual thermal asymmetries has to be considered.
Therefore, in the first step the arithmetic average of all discs is calculated for each direction with equation (22) and (23), wherein $k_{d(z)}$ represents the number of observed discs $d_{(z)}$.

$x_{(z)}=\frac{1}{k_{d(z)}} \cdot \sum_{d(z)} x_{d(z)}$

$y_{(z)}=\frac{1}{k_{d(z)}} \cdot \sum_{d(z)} y_{d(z)}$

In a second step the strict direction-based approach is expanded to a plane-based formulation. The thermal asymmetry vector $\boldsymbol{R}_{(z)}$ for this xy-plane observation along the $z$-axis is calculated with equation (24). The vectors magnitude $\boldsymbol{\tau}_{(z)}=\left|\boldsymbol{R}_{(z)}\right|$ showed to be an appropriate measure for the asymmetry of the entire component. Therefore, the cumulated thermal asymmetry $\boldsymbol{\tau}_{(z)}$ for a component is calculated according to equation (25).

$$
\begin{aligned}
& \boldsymbol{R}_{(z)}=\left(\begin{array}{l}
\frac{1}{k_{d(z)}} \cdot \sum_{d(z)} x_{d(z)} \\
\frac{1}{k_{d(z)}} \cdot \sum_{d(z)} y_{d(z)}
\end{array}\right)=\left(\begin{array}{l}
x_{(z)} \\
y_{(z)}
\end{array}\right) \\
& \boldsymbol{\tau}_{(z)}=\sqrt{x_{(z)}^{2}+y_{(z)}^{2}}
\end{aligned}
$$

\section{MOTOR SPINDLE ASYMMETRY ANALYSIS}

In order to show the potential of the presented method, the thermal asymmetry of motorized spindle was analyzed. Thermal asymmetry optimization can effectively decrease thermal deflection and therefore the radial TCP displacement of motorized spindles. This effect is visualized based on a finite element simulation model of a motorized spindle, which is introduced in chapter 3.1, and its thermal asymmetry optimization in chapter 3.2.

\subsection{Thermal/fluid-mechanical simulation}

The model presented in chapter 3.1 was published in an earlier work of the author team [Koch 2017]. The applied boundary conditions are briefly introduced here to ease the understanding of the following chapter. The boundary conditions are visualized in Fig. 3. It is a coupled thermal/fluid-mechanical simulation model. The electric

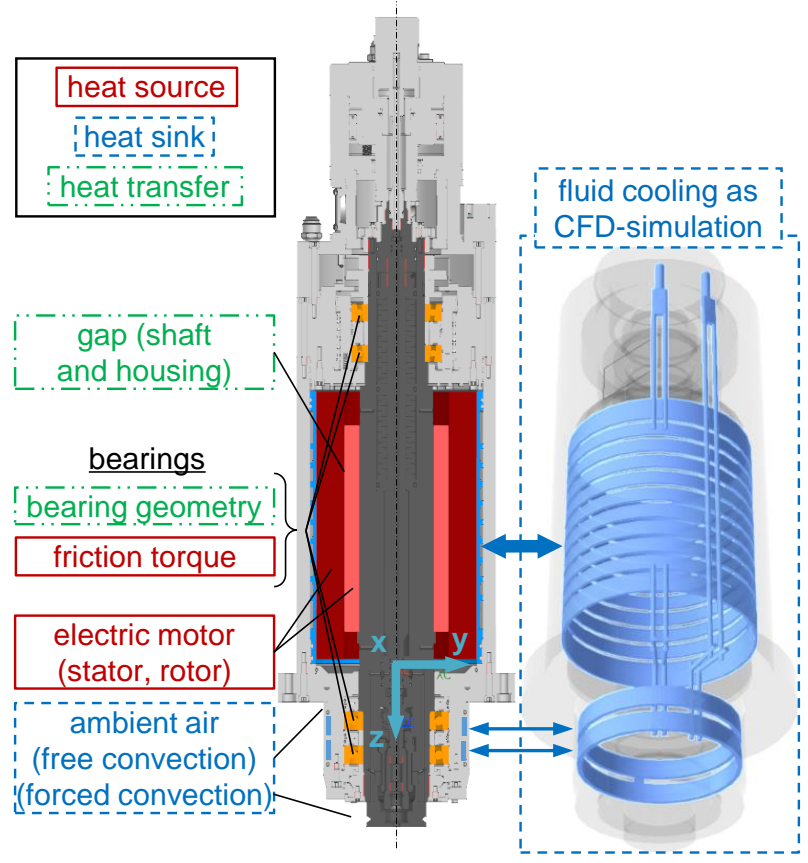

Fig. 3: Boundary condition of the simulation model based on [Koch 2017]. 
motor is considered as heat source, with a separate rotor and stator observation. The four bearings are the second heat source. Both the ambient air and the fluid cooling are considered as heat sinks. The fluid cooling is actually simulated with a computational fluid dynamics simulation. A computational fluid dynamics simulation is essential for this observation, as the fluid cooling itself is the main source of thermal asymmetry in motorized spindles (see chapter 3.2). The heat transfer through the bearing and the heat flux between the shaft and the housing is reflected by appropriate heat transition coefficients.

This set of boundary conditions is comprehensive enough to create a temperature field close to reality. The simulation model was validated based on readings with the real spindle (Fig. 4). Fig. 4 a) represents a thermographic recording of the spindle. It visualizes the spindle after five hours in idle mode at $18.000 \mathrm{rpm}$. Additionally, ten PT-100 sensors were attached to the surface of the spindle. In Fig. 4 b) the simulation model's temperature field is displayed in thermal equilibrium. The heat sources were calculated to reflect the spindle in idle mode at $18.000 \mathrm{rpm}$ in order to establish comparability. The Palmgren formulation for the bearing heat generation was inaccurate. Based on an empirical energy balance analysis its power loss was adjusted. The average temperature deviation between the sensor values and the simulation results was $0,8^{\circ} \mathrm{C}$. All boundary conditions are described in detail in [Koch 2017].

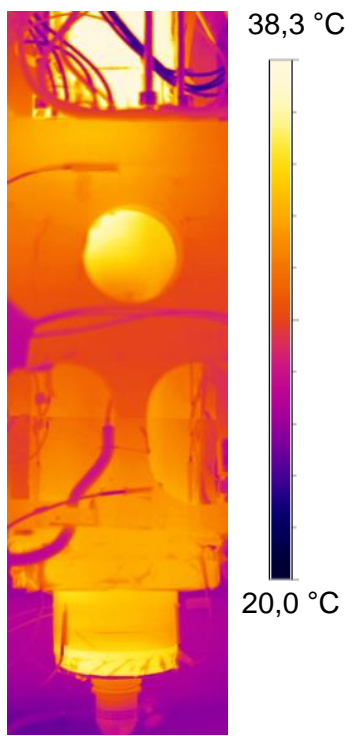

(a) thermographic recording

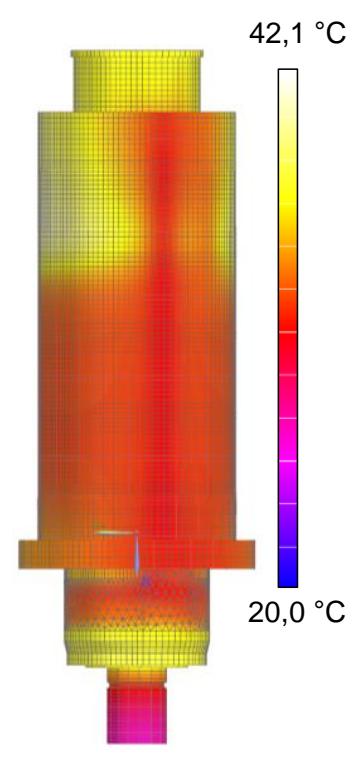

(b) simulation results
Fig. 4: Validation of the spindle simulation model based on [Koch 2017].

\subsection{Radial TCP displacement reduction through thermal asymmetry optimization}

For the validation above, an observation in idle mode with an input power of $815 \mathrm{~W}$ was necessary. Thermal asymmetry rises with increased heat generation. Therefore, the thermal/fluid-mechanical model was adjusted to represent an analysis under load. The motor's power loss was recalculated based on motor slip [Hering 2012] for a nominal input power of $25.000 \mathrm{~W}$.

However, the model described in chapter 3.1 is only capable of calculating an accurate temperature field. In order to simulate the TCP displacement of motorized spindles, an additional thermo-mechanical analysis is

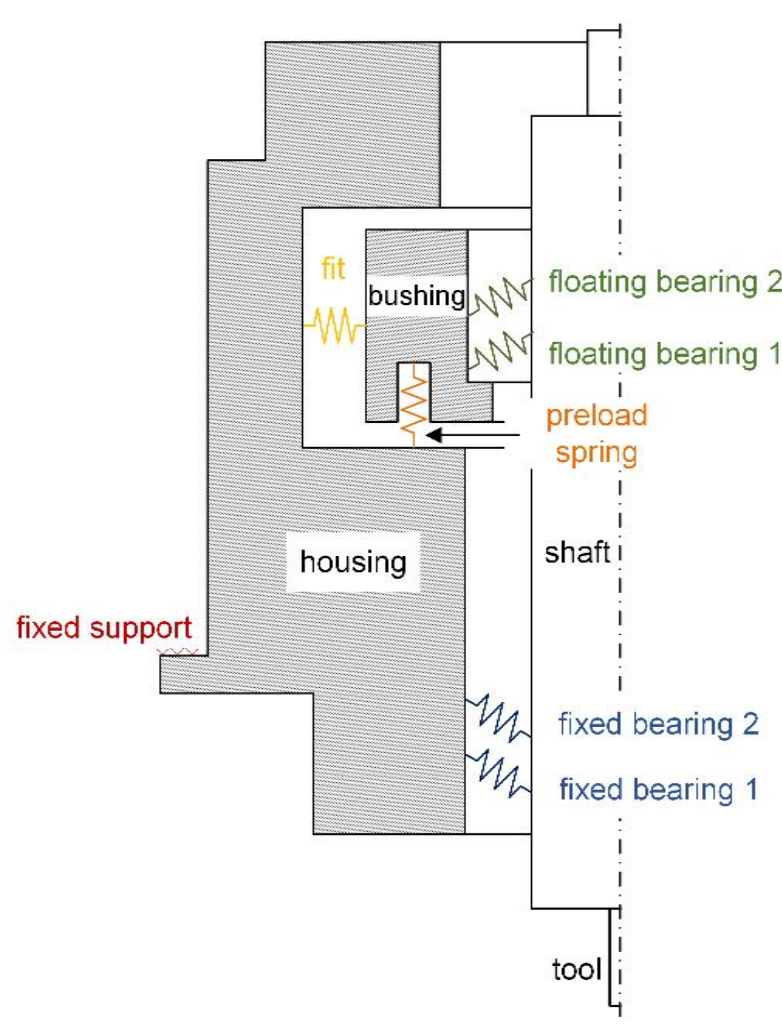

Fig. 5: Thermo-mechanical simulation model of a motorized spindle.

necessary. The temperature field of the thermal/fluidmechanical model is transferred to the thermo-mechanical simulation model in Fig. 5. There is only one load, which is the temperature field itself. Therefore, only the mechanical constraints need to be defined in the thermo-mechanical model. The spindle's bearings are fitted in a tandem arrangement. The bearings are modeled by their respective radial and axial stiffness. The floating bearing itself is mounted in a separate bushing. This bushing allows the thermal expansion of the shaft while keeping the preload almost constant. Both the preload spring and the fit itself are modelled by their respective stiffness. Finally, the

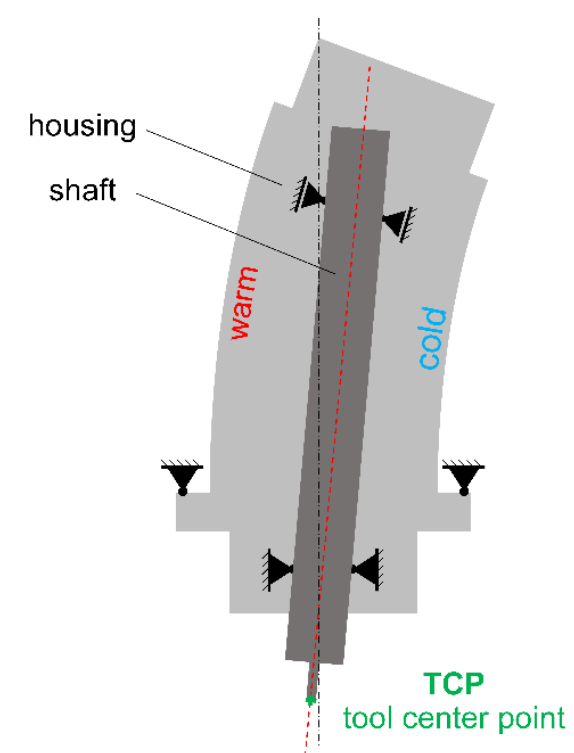

Fig. 6: Radial TCP displacement through thermal asymmetry in the spindles housing. 
surface where the spindle is mounted in the headstock is modelled as fixed support.

The effect on thermal asymmetry in motorized spindles is visualized in Fig. 6. For the radial displacement of the TCP, only the spindle's housing thermal asymmetry is of relevance. The shaft itself actually has no effect on the radial displacement, because its rotation leads to a homogenization of its temperature field. While the fixed support keeps the spindles flange in place, the top part of the housing is thermally deflected because of the fluid cooling system which introduces thermal asymmetry (see Fig. 4). This deflection displaces the floating bearing in the rear accordingly. Consequently, the shaft tips to one side. The TCP is displaced in the opposite direction.

Therefore, a correlation analysis between the spindle housing thermal asymmetry along the z-axis (see Fig 3 ) and the radial TCP displacement should visualize the assumed connection. The housings cumulated thermal asymmetry $\boldsymbol{\tau}_{(z)}$ is calculated according to equation (25) along the z-axis. The current prototype of the algorithm cuts the spindle housing in 20 discs and each of them into four volume elements. The low quantity of volume elements is permissible, as the model features an almost homogenous mesh, but it should definitely be improved in the future. The radial displacement was calculated as the hypotenuse of the TCPs displacement in $x$ - and $y$-direction taken from the simulation's results.

In order to show the assumed connection and the potential of thermal asymmetry as an optimization criterion, a set of seven fluid cooling systems (Fig. 7) in the spindle housing (Fig. 3) was simulated. The design of the fluid cooling system directly influences thermal asymmetry and thereby the radial TCP displacement.

The first system that was analyzed has a circular divided stator cooling sleeve (see Fig. 7 a) and [Weber 2013]). It represents the worst case as it features both the highest thermal asymmetry and the highest radial TCP displacement.

Unlike the circular divided design, the helix stator cooling sleeve in Fig. 7 b) is widely used in practice. In thermal equilibrium it features a radial TCP displacement of $4,75 \mu \mathrm{m}$ in the given configuration. However, it is important to keep in mind that this number increases with tool length. Consequently, the displacement can easily reach between

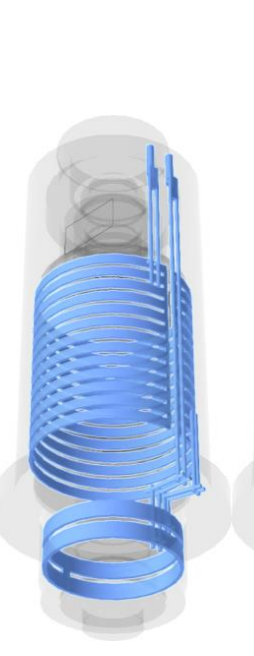

(a) circular divided

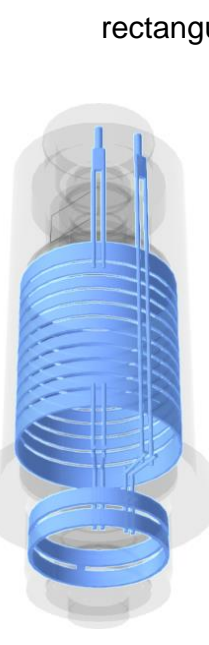

(b) helix

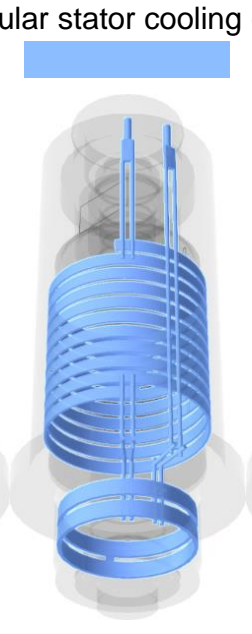

(c) helix without inlet areas

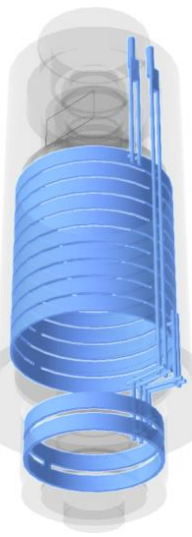

(d) meander

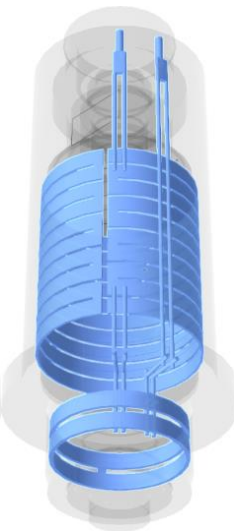

(e) s-meander

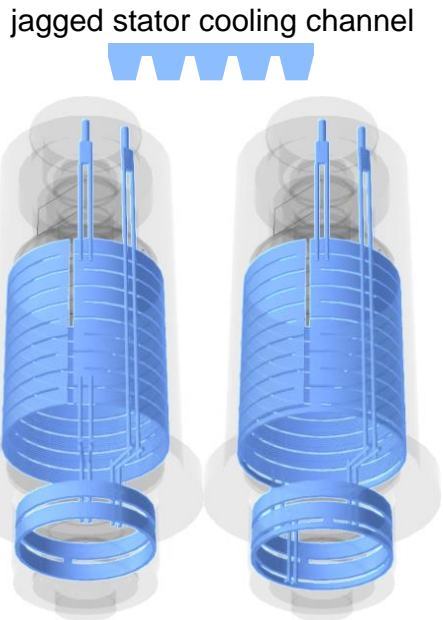

(f) jagged s-meander (g) jagged s-meander modified

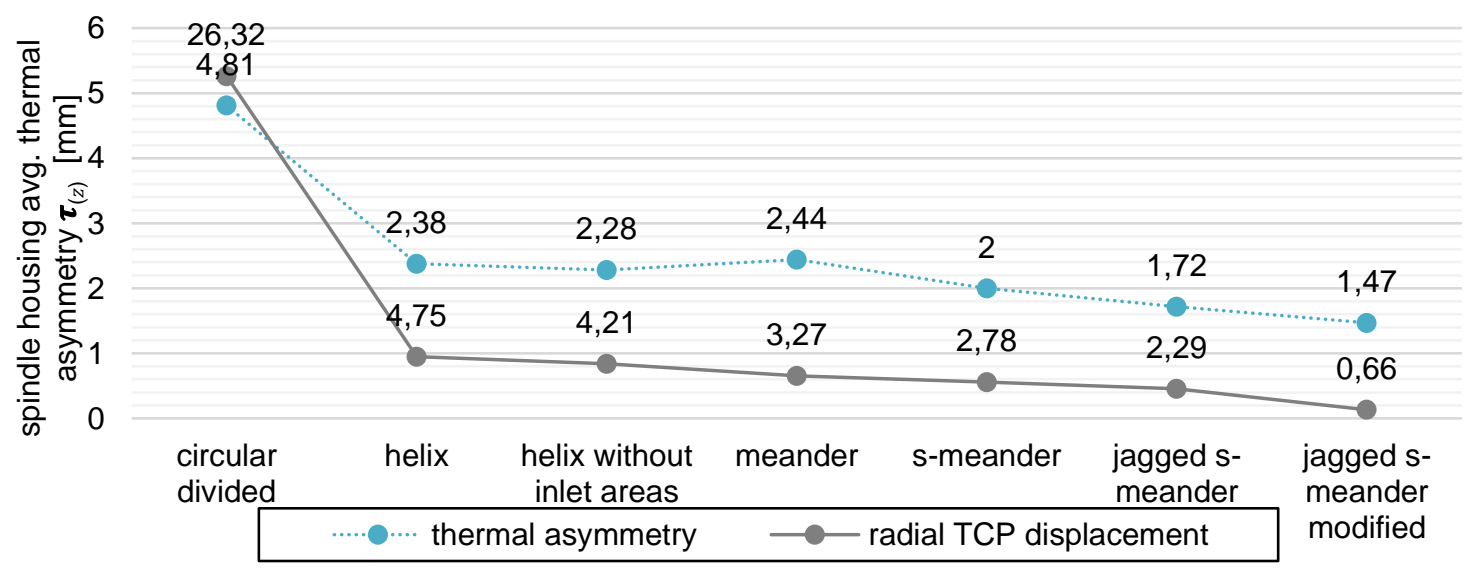

30,00

(h) correlation analysis of the radial TCP displacement and the spindle housing's cumulated thermal asymmetry $\boldsymbol{\tau}_{(z)}$

Fig. 7: Evaluation of seven cooling systems (Fig. 7 a)-g)) to reduce the radial TCP displacement of motorized spindles (Fig. 7 h)). 
10-20 $\mu \mathrm{m}$. For example, a manufacturer measured $15 \mu \mathrm{m}$ radial TCP displacement for a motorized spindle with helix stator cooling sleeve. It is clearly visible that the most widespread cooling concept still has a lot of room for improvement.

The first improvement was an optimization of the inlet areas (Fig. 7 c)). These problematic areas were previously discussed by Weber [Weber 2013]. Deleting them decreased the thermal asymmetry and the radial TCP displacement.

Another design with increasing popularity is the meander shaped stator cooling sleeve in Fig. 7 d). Surprisingly, this design did not decrease the thermal asymmetry, although the radial TCP displacement is improved. This happened most probably, as the simplified kinematical cause-effect relationship in Fig. 6 only applies to the majority of cases. Under certain circumstances, the shaft might show a kink in the area of the fixed bearing. Thus, the thermal deflection problem becomes nonlinear. Possible reasons are extreme thermal asymmetry of the geometry as a whole, or just unfavorable positions of individual highly asymmetric discs.

The next concept of interest is the newly developed smeander in Fig $7 \mathrm{e}$ ). The design of the s-meander is discussed extensively in a paper published simultaneously [Koch 2021]. It features both, a greatly improved thermal asymmetry and radial TCP displacement.

The design's asymmetry and TCP displacement was further improved by jagged fluid channels (Fig. $7 \mathrm{f}$ )) in the area of the stator. This simple modification increases the heat transfer surface. Consequently, the cooling is improved which surprisingly decreases thermal asymmetry as well.

So far, the spindle manufacturers assumed that the main cause of thermal asymmetry is the stator cooling sleeve. The stator cooling sleeve easily absorbs the largest amount of heat. However, the drill network which leads the cooling fluid from the top of the spindle to the front bearings, back to the stator, through the cooling sleeve and out of the system, is of no less importance for the thermal asymmetry. A spindle with a modified drill network is visualized in Fig. $7 \mathrm{~g}$ ). The drill holes coming from the front bearings were relocated to the opposite side of the spindle. This decreases the thermal asymmetry significantly. By reducing the spindle housing's cumulated thermal asymmetry to 1,47 $\mathrm{mm}$, the radial TCP displacement can be reduced to $660 \mathrm{~nm}$.

\section{SUMMARY}

Thermal displacement of the tool center point (TCP) is the main reason for decreased manufacturing precision in machine tools. Therefore, multiple research activities aim to decrease thermal expansion and deflection issues through different complex compensation approaches. In contrast to these concepts, this paper introduced a method to identify the causes of thermal deflection, thereby giving the ability to eliminate them in the construction phase.

As thermal asymmetry is the cause of thermal deflection, chapter 2 introduces a new method with the aim to quantify thermal asymmetry along an axis. The formulation is applied to finite element simulation models, as those usually feature detailed temperature fields. The method requires a separate discretization of the observed component to cluster its geometry in discs and volume elements. The observed component needs to be quantified geometrically and thermally. The geometrical computation is based on a centroid calculation for each disc. Comparably, the temperature fields constitution is described by a newly introduced thermal centroid quantification. The paper's target value, thermal asymmetry, is defined as the difference of thermal and geometrical centroid locations for each disc along an axis. For a comprehensive analysis, the approach was extended by an additional calculation strategy to quantify the thermal asymmetry of entire components.

Chapter 3 shows the practicability of the calculation method though its application to motorized spindles. An already published, validated simulation model of a motorized spindle was used as data basis. The cause-effect relation of thermal asymmetry and the radial TCP displacement was analyzed and revealed the spindle housing's asymmetry to be the relevant problem. This asymmetry is caused by the fluid cooling system. Subsequently, the fluid passages were optimized in seven constructional adjustments. Decreasing the thermal asymmetry of the motorized spindle lowers the radial TCP displacement and therefore confirms their correlation. The radial TCP displacement was reduced by $97,5 \%$ solely with constructive measures.

In conclusion, the presented formulation is a capable approach to quantify thermal asymmetry. Tackling this issue in the construction phase solves the issue of thermal deflection with little effort. The algorithm's applicability is not restricted to motorized spindles and it could be applied to other components in the future.

\section{REFERENCES}

[Abele 2010] Abele, E. et al. Machine tool spindle units, CIRP Annals, 2010, Volume 59, Issue 2, pp 781-802. ISSN 0007-8506

[Baum 2018] Baum, C. Thermally induced volumetric error compensation by means of integral deformation sensors, Procedia CIRP, 2018, Volume 72, pp 1148-1153. ISSN 2212-8271

[Beitelschmidt 2015] Beitelschmidt, M. et al. Innovative simulation technology for real-time calculation of the thermo-elastic behavior of machine tools in motion, Applied Mechanics and Materials, 2015, volume 794, pp 363-370

[Brecher 2009] Brecher, C. and Wissmann, A. Optimisation of thermal behaviour of milling machines, ZWF Zeitschrift fuer wirtschaftlichen Fabrikbetrieb, 2009, Volume 104, pp 437-441. ISSN: 0947-0085

[Brecher 2014] C. Brecher et al. Temperaturstabile Werkzeugmaschinen: Messverfahren zur volumetrischen Korrektur thermoelastischer Verlagerungen, wt Werkstattstechnik online, 2014, Volume 104, pp 490-495. ISSN 00432806

[Brecher 2015] Brecher, C. et al. Metrological analysis of a mechatronic system based on novel deformation sensors for thermal issues in machine tools, Procedia CIRP, 2018, Volume 77, pp 517-520. ISSN 2212-8271

[Brecher 2017] Brecher, C. et al. Analysis of spatial and temporal dependencies of the TCP-dislocation measurement for the assessment of the thermo-elastic behavior of 3-axis machine tools, In: Blunt, Liam, ed., 12th International Conference and Exhibition on Laser Metrology, Machine Tool, CMM \& Robotic Performance; Lamdamap, 15-16 March 2017, Wotton-under-Edge (UK), Gloucestershire Bedfordshire: Euspen, pp 122-132. ISBN 978-0-95667909-3

[Brecher 2018] Brecher, C. et al. Metrological analysis of a mechatronic system based on novel deformation sensors for thermal issues in machine tools, Procedia CIRP, 2018, Volume 77, pp 517-520, ISSN 2212-8271 
[Fetzer 1999] Fetzer, A and Fraenkel, H. Mathematik 2, Lehrbuch fuer ingenieurwissenschaftliche Studiengänge, 1999, volume 5, Berlin Heidelberg: Springer, ISBN 978-3540-65584-8

[Grossmann 2006] Grossmann, $K$ and Jungnickel, $G$. Prozessgerechte Bewertung des thermischen Verhaltens von Werkzeugmaschinen, Inst. fuer Werkzeugmaschinen und Steuerungstechnik, Lehrstuhl fuer Werkzeugmaschinen, Dresden, 2006. ISBN 3-86005-547$\mathrm{X}$

[Grossmann 2015] Grossmann, K., ed., Thermo-energetic Design of Machine Tools, 2015, Basel: Springer International Publishing. ISBN 978-3-319-12624-1

[Hering 2012] Hering, E. et al. Elektrotechnik und Elektronik für Maschinenbauer, 2012, Berlin Heidelberg: Springer. ISBN 978-3-642-12881-3

[Horejs 2012] Horejs, O. et al. Advanced Modelling of Thermally Induced Displacements and Its Implementation into Standard CNC Controller of Horizontal Milling Center, Procedia CIRP, 2012, Volume 4, pp 67-72. ISSN 22128271

[Koch 2017] Koch, L. et al. Coupled thermal and fluid mechanical modeling of a high speed motor spindle, Energy Efficiency in Strategy of Sustainable Production III, 2017, Volume 871, Applied Mechanics and Materials, Trans Tech Publications Ltd, pp 161-168

[Koch 2021] Koch, L. et al. Comparative Analysis of Fluid Cooling Systems in Motorized Spindles, In: M. Sulitka and S. Ihlenfeldt, ed. proceedings of the Conference on Thermal Issues in Machine Tools: Prague, April 19-21, 2021

[Liu 2016] Liu, K. et al. Modeling and compensation for spindle's radial thermal drift error on a vertical machining center, International Journal of Machine Tools and Manufacture, 2016, Volume 105, pp 58-67. ISSN 08906955

[Mayr 2012] Mayr, J. et al. Thermal issues in machine tools, CIRP Annals, 2012, Volume 61, Issue 2, pp 771-791, ISSN 0007-8506
[Naumann 2016] Naumann, C. et al. Characteristic Diagram Based Correction Algorithms for the Thermo-elastic Deformation of Machine Tools, Procedia CIRP, Volume 41, 2016, pp 801-805, ISSN 2212-8271

[Putz 2016] Putz, M. et al. Implementation and demonstration of characteristic diagram as well as structure model based correction of thermo-elastic tool center point displacements, Journal of Machine Engineering, 2016, Volume 16, pp 88-101. ISSN 1895-7595

[Putz 2018] Putz, M. et al. Industrial relevance and causes of thermal issues in machine tools, In: S. Ihlenfeldt et al., ed. proceedings of the Conference on Thermal Issues in Machine Tools: Dresden, 2018, Wissenschaftliche Scripten, pp 127-139. ISBN 9783957350855

[Putz 2019] Putz, M. et al. Thermal errors in milling: Comparison of displacements of the machine tool, tool and workpiece, Procedia CIRP, 2019, Volume 82, pp 389-394. ISSN 2212-8271

[Thiem 2016] Thiem, X. et al. Principle and Verification of a Structure Model Based Correction Approach, Procedia CIRP, 2016, Volume 46, pp 111-114. ISSN 2212-8271

[Thiem 2017] Thiem, X. et al. Structure Model Based Correction of Thermally Induced Motion Errors of Machine Tools, Procedia Manufacturing, 2017, Volume 14, pp 128135. ISSN 2351-9789

[Weber 2013] Weber, J. and Weber, J. Thermo-energetic analysis and simulation of the fluidic cooling system of motorized high-speed spindles, proceedings of the Scandinavian International Conference on Fluid Power, SICFP2013, Linköping, June 3-5, 2013

[Weck 1995] Weck, M. et al. Reduction and Compensation of Thermal Errors in Machine Tools, CIRP Annals, 1995, Volume 44, Issue 2, pp 589-598. ISSN 0007-8506

[Zhang 2018] Zhang, Y. et al., Active and intelligent control onto thermal behaviors of a motorized spindle unit, The International Journal of Advanced Manufacturing Technology, 2018, Volume 98, pp 3133-3146 